\title{
Crystal Structure of a Hydrolyzed Product of the Cobalt(III) Complex with 1-(3,5-Dichlorosalicylideneamino)-3-amino-2-propanol
}

\author{
Masahiro Mikuriya, ${ }^{* \dagger}$ Nagisa Tsuchimoto,* Yoshiki Koyama,* Ryoji Mitsuhashi, ${ }^{* *}$ and \\ Motohiro TsuBOI* \\ *Department of Applied Chemistry for Environment and Research Center for Coordination Molecule-based \\ Devices, School of Biological and Environmental Sciences, Kwansei Gakuin University, 2-1 Gakuen, \\ Sanda 669-1337, Japan \\ **Institute of Liberal Arts and Science, Kanazawa University, Kakuma, Kanazawa 920-1192, Japan
}

\begin{abstract}
The crystal structure of a hydrolyzed product of the cobalt(III) complex with 1-(3,5-dichlorosalicylideneamino)-3-amino2-propanol $\left(\mathrm{H}_{2} \mathrm{dcsalapr}\right)$ was determined by the single-crystal X-ray diffraction method at $90 \mathrm{~K}$. It crystallizes in the triclinic space group $P \overline{1}$ with $a=9.2242(15) \AA, b=9.3391(16) \AA, c=9.5525(16) \AA, \alpha=75.574(3)^{\circ}, \beta=85.550(3)^{\circ}, \gamma=$ $69.154(3)^{\circ}, V=744.7(2) \AA^{3}, D_{\mathrm{x}}=1.634 \mathrm{~g} / \mathrm{cm}^{3}$, and $Z=1$. The $R 1[I>2 \sigma(I)]$ and $w R 2$ (all data) values are 0.0489 and 0.1093 , respectively, for all 3319 independent reflections. The X-ray structure analysis revealed a centrosymmetric octahedral Co(III) complex with two hydrolyzed Schiff-base ligands, Hdcsalapr-, in a facial mode, one chloride ion, two acetonitrile molecules, and two water molecules in the unit cell, which was formed from the reaction solution of cobalt(II) chloride hexahydrate with 1,3-bis(3,5-dichlorosalicylideneamino)-2-propanol in the presence of triethylamine in acetonitrile.
\end{abstract}

(Received August 31, 2021; Accepted September 13, 2021; Published on web January 10, 2022)

\begin{abstract}
Much interest has been attracted to the coordination chemistry of potential pentadentate Schiff-base ligands, 1,3-bis(salicylideneamino)-2-propanol $\left(\mathrm{H}_{3}\right.$ bsalpr) and its substituted derivatives, with two phenolato-oxygen, two imino-nitrogen, and one alcoholate-oxygen donor atoms, are of interest. In the crystals, these Schiff-base ligands have intramolecular hydrogen bonds between imino-nitrogen and phenolato-oxygen atoms and intermolecular hydrogen bonds between an alcohol-oxygen atom and a phenolato-oxygen atom of neighboring Schiff-base molecule. ${ }^{1-5}$ The reaction between these Schiff-base ligands and metal salts generates mononuclear copper(II), ${ }^{6}$ tetranuclear zinc(II) ${ }^{7}$ complexes with a tetradentate $O, N, N, O$-chelate, and dinuclear copper(II), ${ }^{8}$ nickel(II), ${ }^{9}$ manganese(III), ${ }^{10,11}$ and cobalt(III) ${ }^{12}$, and tetranuclear manganese(II,III) ${ }^{13,14}$ complexes with a tridentate $O, N, O$-chelate. Aiming at extending these systems, we decided to prepare a cobalt complex with 1,3-bis(3,5-dichlorosalicylideneamino)-2-propanol ( $\mathrm{H}_{3}$ bdcsalpr), which has not been reported so far. In this study, we performed a reaction between $\mathrm{H}_{3}$ bdcsalpr and cobalt(II) chloride hexahydrate in the presence of triethylamine in acetonitrile, and determined the crystal structure of the isolated complex, which has been found to be a new mononuclear cobalt(III) complex with hydrolyzed Schiff-base ligands, 1-(3,5-dichlorosalicylideneamino)-3-amino-2-propanol (Hdcsalapr-), [Co(Hdcsalapr $\left.)_{2}\right]$ $\mathrm{Cl} \cdot 2 \mathrm{CH}_{3} \mathrm{CN} \cdot 2 \mathrm{H}_{2} \mathrm{O}(\mathbf{1})$, as depicted in Fig. 1, is different from the expected dinuclear cobalt complexes that we prepared before. ${ }^{12}$

A Schiff-base ligand, $\mathrm{H}_{3}$ bdcsalpr, was synthesized by a similar method to the previously reported one. ${ }^{5}$ 1,3-Diamino-2propanol $(0.703 \mathrm{~g}, 7.8 \mathrm{mmol})$ and 3,5-dichlorosalicylaldehyde $(3.00 \mathrm{~g}, 15.7 \mathrm{mmol})$ were dissolved in methanol $\left(70 \mathrm{~cm}^{3}\right)$. The
\end{abstract}

$\dagger$ To whom correspondence should be addressed.

E-mail: junpei@kwansei.ac.jp solution was refluxed for $3 \mathrm{~h}$, and then left at room temperature. The resulting yellow precipitate was filtered off, washed with methanol, and dried under a vacuum. Yield, $3.16 \mathrm{~g}(93 \%)$. IR $\left(\mathrm{KBr}, \quad \mathrm{cm}^{-1}\right): 3219(v(\mathrm{OH})), 3064 \quad(v(\mathrm{CH}($ phenyl $)), 2951$ $\left(v_{\mathrm{as}} \mathrm{CH}_{2}\right), 2872\left(v_{\mathrm{s}} \mathrm{CH}_{2}\right), 1626(v \mathrm{C}=\mathrm{N})$.

Single crystals of 1 were obtained as follows: $\mathrm{H}_{3}$ bdcsalpr (42.5 mg, $0.1 \mathrm{mmol}$ ) was dissolved in acetonitrile $(3 \mathrm{~mL})$. To this solution, cobalt(II) chloride hexahydrate $(24.0 \mathrm{mg}$, $0.1 \mathrm{mmol}$ ) and six drops of triethylamine were added. The resulting brown solution was filtered after standing for several days to remove a small amount of white precipitate, and then the filtrate was left at room temperature for a further several days to give reddish-brown crystals. Yield, $5.7 \mathrm{mg}$ (7.8\% based on the metal salt). Reflectance spectra: $\lambda_{\max } 284,422$ $\left({ }^{1} A_{1 \mathrm{~g}} \rightarrow{ }^{1} T_{2 \mathrm{~g}}\right), 550 \mathrm{sh}\left({ }^{1} A_{1 \mathrm{~g}} \rightarrow{ }^{1} T_{1 \mathrm{~g}}\right), 724\left({ }^{1} A_{1 \mathrm{~g}} \rightarrow{ }^{3} T_{2 \mathrm{~g}}\right) \mathrm{nm}$.

X-ray diffraction data were collected on a Bruker CCD X-ray diffractometer (SMART APEX) using graphite-monochromated Mo- $K \alpha$ radiation. Crystal data and details concerning the data collection are given in Table 1 . The structure was solved by an

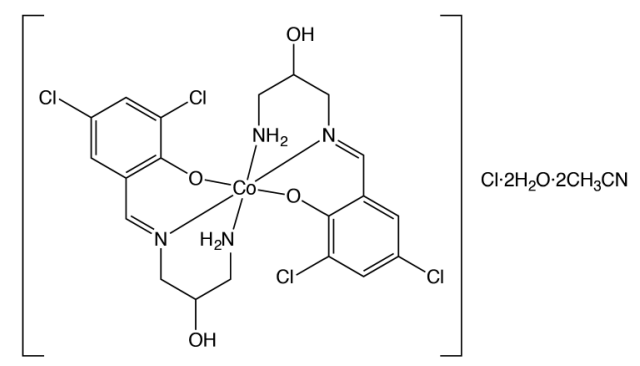

Fig. 1 Chemical structure of the title compound. 
Table 1 Crystal and experimental data

Chemical formula: $\mathrm{C}_{24} \mathrm{H}_{28} \mathrm{Cl}_{5} \mathrm{CoN}_{6} \mathrm{O}_{6}$

Formula weight $=732.70$

$T=90 \mathrm{~K}$

Crystal system: triclinic

Space group: $P \overline{1}$

$a=9.2242(15) \AA \quad \alpha=75.574(3)^{\circ}$

$b=9.3391(16) \AA \quad \beta=85.550(3)^{\circ}$

$c=9.5525(16) \AA \quad \gamma=69.154(3)^{\circ}$

$V=744.7(2) \AA^{3}$

$Z=1$

$D_{\text {x }}=1.634 \mathrm{~g} / \mathrm{cm}^{3}$

Radiation: Mo $K \alpha(\lambda=0.71073 \AA)$

$\mu(\operatorname{Mo} K \alpha)=1.075 \mathrm{~mm}^{-1}$

$F\left(\begin{array}{lll}0 & 0 & 0\end{array}\right)=374$

Crystal size $=032 \times 0.12 \times 0.10 \mathrm{~mm}^{3}$

No. of reflections collected $=4689$

No. of independent reflections $=3319$

$\theta$ range for data collection: 2.363 to $27.484^{\circ}$

Data/Restraints/Parameters $=3319 / 0 / 203$

Goodness-of-fit on $F^{2}=0.953$

$R$ indices $[I>2 \sigma(I)]: R 1=0.0489, w R 2=0.1023$

$R$ indices (all data): $R 1=0.0786, w R 2=0.1093$

$(\Delta / \sigma)_{\max }=0.000$

$(\Delta \rho)_{\max }=0.665 \mathrm{e}^{-3}$

$(\Delta \rho)_{\min }=-0.452 \mathrm{e}^{-3}$

Measurement: Bruker Smart APEX CCD diffractometer

Program system: SHELXTL

Structure determination: intrinsic phasing (SHELXT-2014/5)

Refinement: full matrix least-squares (SHELXL-2018/3)

CCDC deposition number: 2104534

intrinsic phasing method with SHELXT-2014, and refined by full-matrix least-squares methods with SHELXL-2018. The hydrogen atoms attached to the amino-nitrogen atom (N2) were located from the D-Fourier map, whereas the hydrogen atoms of the water-oxygen atom $(\mathrm{O} 3)$ could not be found by the D-Fourier map; the other hydrogen atoms were inserted at their calculated positions, and fixed there. Crystallographic data have been deposited with Cambridge Crystallographic Data Centre (Deposit number CCDC-2104534). Copies of the data can be obtained free of charge via http://www.ccdc.cam.ac.uk/conts/ retrieving.html (or from the Cambridge Crystallographic Data Centre, 12, Union Road, Cambridge, CB2 1EZ, UK; Fax, +44 1223 336033; e-mail,deposit@ccdc.cam.ac.uk).

The X-ray structure analysis of $\mathbf{1}$ revealed that the unit cell consists of a centrosymmetric octahedral Co(III) complex cation with two hydrolyzed Schiff-base ligands, 1-(3,5-dichlorosalicylideneamino)-3-amino-2-propanol (Hdcsalapr), centered at the $\mathrm{Co} 1$ atom, $\left[\mathrm{Co}\left(\mathrm{Hdcsalapr}_{2}\right]^{+}\right.$, one chloride ion $(\mathrm{Cl} 3)$, two acetonitrile molecules, and two water molecules. The Col and $\mathrm{Cl} 3$ atoms are located at the crystallographic inversion centers. An ORTEP drawing of the [Co(Hdcsalapr $\left.)_{2}\right]$ complex cation is shown in Fig. 2. Selected bond distances and angles are given in Table 2. The Co1 atom is coordinated by two sets of the phenolate $\mathrm{O} 1$, imino $\mathrm{N} 1$, and amino N2 atoms of the Hdcsalapr ligand in a facial mode to achieve an octahedral geometry. The Co1-O1, Co1-N1, and Co1-N2 distances are 1.903(2), 1.924(3), $1.962(3) \AA$, respectively, being normal as $\mathrm{Co}(\mathrm{III})-\mathrm{O}$ and $\mathrm{N}$ bond distances. ${ }^{15}$ The Hdcsalapr ligand forms two adjacent sixmembered chelate rings with the Co1 atom, where the Co1-O1C1-C6-C7-N1 ring forms a nearly planar configuration, whereas the Co1-N1-C8-C9-C10-N2 ring takes a chair conformation. In the crystal, the alcohol oxygen atom $\mathrm{O} 2$ is hydrogen-bonded to a water molecule (O3), which is further hydrogen-bonded by

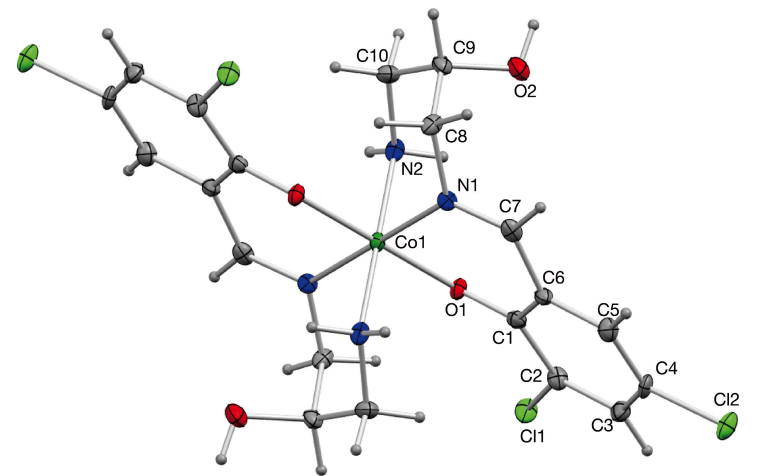

Fig. 2 Complex cation, $\left[\mathrm{Co}(\mathrm{Hdcsalapr})_{2}\right]^{+}$, of the title compound. The thermal ellipsoids are shown at the $50 \%$ probability level.

Table 2 Selected bond distances $(\AA)$ and angles $\left({ }^{\circ}\right)$

\begin{tabular}{llll}
\hline Co1-O1 & $1.903(2)$ & N1-C7 & $1.285(4)$ \\
Co1-N1 & $1.924(3)$ & N1-C8 & $1.466(4)$ \\
Co1-N2 & $1.962(3)$ & N2-C10 & $1.497(4)$ \\
O1-Co1-O1 & 180.0 & N1-Co1-N2 & $87.20(12)$ \\
O1-Co1-N1 & $90.75(10)$ & N1-Co1-N2 & $92.80(12)$ \\
O1-Co1-N1 & $89.25(10)$ & N1-Co1-N1 & 180.0 \\
O1-Co1-N2 & $88.23(11)$ & N2-Co1-N2 & 180.0 \\
O1-Co1-N2 & $91.77(11)$ & & \\
\hline
\end{tabular}

Symmetry code: i $(1-x,-y, 1-z)$.

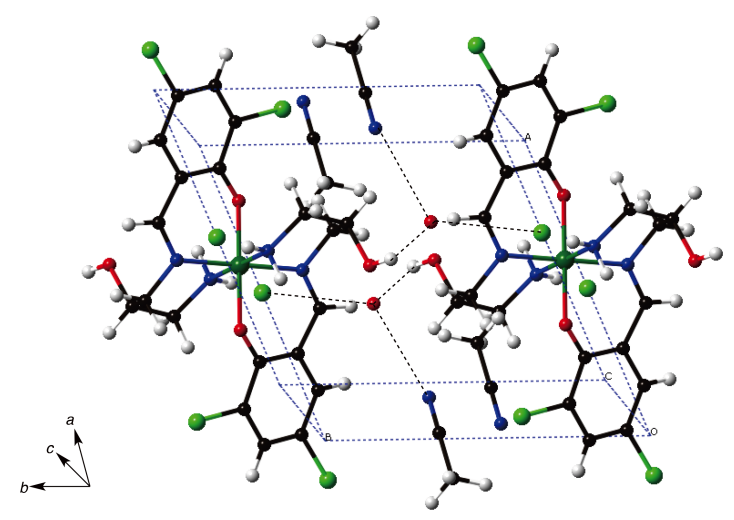

Fig. 3 Packing diagram of the title compound, showing hydrogen bonds as dashed lines.

intermolecular hydrogen bonds with a chloride ion $(\mathrm{Cl} 3)$ and an

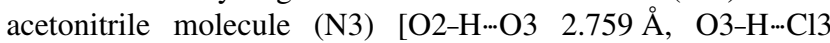
$3.211 \AA$, O3-H...N3 $3.116 \AA$ ] (Fig. 3).

The diffused reflectance spectra of $\mathbf{1}$ (Fig. 4) are characteristic of octahedral low-spin cobalt(III), showing d-d bands in the visible region, being in harmony with the octahedral geometry of $\left[\mathrm{Co}(\mathrm{Hdcsalapr})_{2}\right]^{+}$. The spectra are similar to those of the dinuclear octahedral cobalt(III) complexes with 1,3-bis(3nitrosalicylideneamino)-2-propanolato and 1,3-bis(5-nitrosalicylideneamino)-2-propanolato ligands. ${ }^{12}$ The infrared spectra of $\mathrm{H}_{3}$ bdcsalpr (Fig. 5) shows the absence of $v_{\mathrm{as}} \mathrm{NH}_{2}$ and $v_{\mathrm{s}} \mathrm{NH}_{2}$ bands expected to appear at $\sim 3560$ and $\sim 3400 \mathrm{~cm}^{-1}$, respectively, ${ }^{16}$ excluding the possibility of hydrolyzation of the Schiff-base ligand before the reaction with the metal ion. Therefore, the formation of a hydrolyzed Schiff-base in the 


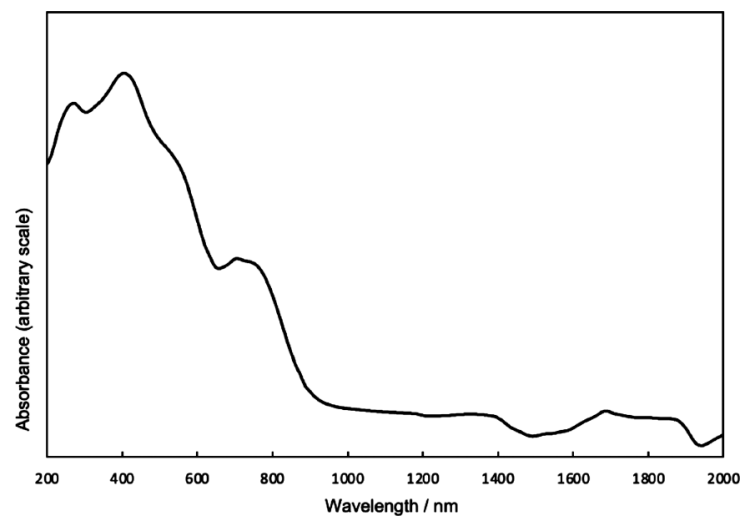

Fig. 4 Diffused reflectance spectra of the title compound.

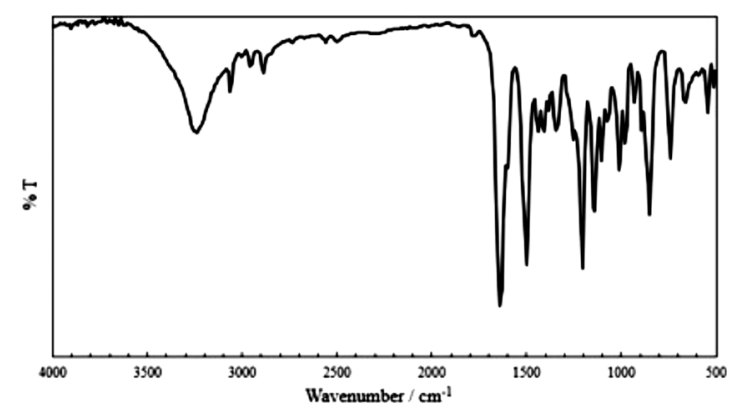

Fig. 5 Infrared spectra of $\mathrm{H}_{3}$ bdcsalpr ( $\mathrm{KBr}$ disk).

present complex may have occurred by a small amount of water molecules originated from metal salt and/or an acetonitrile solvent in the presence of triethylamine. A similar hydrolysis reaction and the degradation of Schiff-base molecules were observed in a few of metal complexes with Schiff-base ligands. ${ }^{10,17,18}$

\section{Acknowledgements}

The present work was partially supported by Grants-in-Aid for Scientific Research No. 17K05820 from the Ministry of Education, Culture, Sports, Science and Technology and the MEXT-Supported Program for the Strategic Research Foundation at Private Universities, 2010 - 2014.

\section{References}

1. M. Azam, Z. Hussain, I. Warad, S. I. Al-Resayes, M. S. Khan, M. Shakir, A. Trzesowska-Kruszynska, and R. Kruszynski, Dalton Trans., 2012, 41, 10854.

2. M. Mikuriya, Y. Naka, and D. Yoshioka, X-ray Struct. Anal. Online, 2015, 31, 55. Erratum: X-ray Struct. Anal. Online, 2016, 32, 11.

3. M. T. Kaczmarek, M. Skrobanska, M. Zabiszak, M. WalesaChorab, M. Kubicki, and R. Jastrzab, RSC Adv., 2018, 8, 30994.

4. M. Mikuriya, Y. Koyama, and R. Mitsuhashi, X-ray Struct. Anal. Online, 2019, 35, 33.

5. M. Mikuriya, N. Tsuchimoto, Y. Koyama, R. Mitsuhashi, and M. Tsuboi, X-ray Struct. Anal. Online, 2022, 38, 3.

6. E. Donmez, H. Kara, A. Karakaş, H. Ünver, and A. Elmali, Spectrochim. Acta, 2007, A66, 1141.

7. M. Mikuriya, S. Ikenoue, R. Nukada, and J.-W. Lim, Bull. Chem. Soc. Jpn., 2001, 74, 101.

8. Y. Nishida and S. Kida, J. Chem. Soc., Dalton Trans., 1986, 2633.

9. M. Mikuriya, T. Sasaki, A. Anjiki, S. Ikenoue, and T. Tokii, Bull. Chem. Soc. Jpn., 1992, 65, 334.

10. M. Mikuriya, Y. Yamato, and T. Tokii, Bull. Chem. Soc. Jpn., 1992, 65, 1466.

11. M. Mikuriya, Y. Koyama, D. Yoshioka, and R. Mitsuhashi, X-ray Struct. Anal. Online, 2020, 36, 7.

12. M. Mikuriya, Y. Naka, D. Yoshioka, and M. Handa, X-ray Struct. Anal. Online, 2016, 32, 55.

13. M. Mikuriya, S. Kudo, C. Matsumoto, S. Kurahashi, S. Tomohara, Y. Koyama, D. Yoshioka, and R. Mitsuhashi, Chem. Papers, 2018, 72, 853.

14. M. Mikuriya, S. Kurahashi, S. Tomohara, Y. Koyama, D. Yoshioka, R. Mitsuhashi, and H. Sakiyama, Magnetochemistry, 2019, 5, 8 .

15. R. Grobelny, M. Melniik, and J. Mrozinski, "Cobalt Coordination Compounds: Classification and Analysis of Crystallographic and Structural Data", 1996, Dolnoslaskie Wydawnictwo Edukacyjne, Wroclaw.

16. K. Nakanishi, P. H. Solomon, and N. Furutachi, "Infrared Absorption Spectroscopy”, 1978, Nankodo, Tokyo.

17. M. Mikuriya, K. Nakadera, and T. Tokii, Inorg. Chim. Acta, 1992, 194, 129.

18. M. Mikuriya, S. Ono, Y. Koyama, R. Mitsuhashi, and M. Tsuboi, X-ray Struct. Anal. Online, 2021, 37, 41. 